\title{
Evolutionary Algorithm for Scheduling in Wireless Sensor Networks
}

\author{
Yaser Khamayseh ${ }^{1}$, Wail Mardini ${ }^{1}$, Nadhir Ben Halima ${ }^{2}$ \\ ${ }^{1}$ Department of Computer Science, Jordan University of Science and Technology, Irbid - Jordan, Canadian. \\ 2 Department of Computer Science, Taibah University, Yanbu - KSA. \\ * Corresponding author. Tel.: +962779060324; email: yaser@just.edu.jo \\ Manuscript submitted March 10, 2017; accepted June 8, 2017. \\ doi: $10.17706 /$ jcp.13.3.262-270
}

\begin{abstract}
Sensor networks are a collection of sensor nodes spread in a geographical location. Nodes collect information and send this information to a sink node (access point). They usually form many-to-one sensor network where all traffic generated at sensors is destined for AP, thus a routing tree is formed. Scheduling is the process of deciding which node to send at a particular time. TDMA scheduling have been studied in terms of minimizing packet delay, improving fairness, maximizing parallel operation, minimizing the energy consumption, and shortening the total slots to finish a set of transmission tasks. Allowing the sensors to turn their radio off when not active is a common energy-saving strategy. Intelligent search algorithms were used to obtain an efficient scheduler

In this work we look at the usage of 2 particular algorithms: evolutionary (EA) and particle swarm optimization (PSO). Then, we propose a hybrid algorithm that utilizes both EA and PSO algorithms using different optimization functions. The performance of the propped algorithm is evaluated using simulation. The obtained simulation results demonstrated that the PSO different optimization functions will give different fitness values and results.
\end{abstract}

Key words: WSN, energy, TDMA, scheduling, particle swarm optimization (PSO), evolutionary algorithm (EA).

\section{Introduction}

Wireless network is a significant and widely used technology that allows the user to communicate with each other using radio waves instead of using network cables. Wireless networks are usually categorized into three types; Cellular, Ad hoc, and sensor network. Cellular networks, through a central access points called base stations, are usually used to provide voice service to wide range of users, modern cellular systems (such as UMTS) provides internet access as well as advanced data communications services. Ad hoc networks provide various applications in many real-life scenarios that lack a central access point, such commercial, defense, pervasive and scientific applications. Finally, sensor network interact with its environment by using special equipment for sensing and actuating.

Wireless network has the capability of self-organization, self-configuring by encapsulating the details from end users, less expensive, convenient, disaster recovery and deployed anywhere. Despite of these capabilities, it meets some limitations such as limited range of radio waves, meets interference problem, power consumption, need for special devices, and security. Moreover, to provide the above mentioned 
capabilities without the existence of a central point, the design of such networks is a challenging task. Sensor nodes suffers mainly of the limited resources, such as power, it becomes very difficult to estimate the available resources in the network in an efficient way, moreover, it is more challenging to distribute such resources fairly among the nodes. Thus, scheduling is a challenging problem in sensor networks.

Scheduling in wireless network defined of how to commit resources between varieties of possible tasks. Moreover, presents a great challenge for provisioning bandwidth, maximizing network performance and minimizing the number of collided transmission. Therefore, TDMA MAC protocol is a good choice for satisfying some parts of these challenges, which is, divide the time into slots and assign each packet of data to transmit at the beginning of specific slot.

The problem defines a number of distributed sensor networks and an access point; a sensor network represented by an undirected graph $G=(V, E)$, where $V$ represents the set of all nodes in the network and $\mathrm{E}$ represents the set of communication links between nodes. The function $d(i, j)$ represents the minimum number of edges to travel from node $i$ to node $j$ (distance function).

Moreover, an interference matrix IN*N describes the conflict relationship in the network such that

$$
\begin{gathered}
\text { If } d(i, j) \leq 2 \\
I_{i j}=1 ; \\
\text { Else } \\
\quad I_{i j}=0
\end{gathered}
$$

Hence, if the distance $d(i, j)>2$, then nodes $i$ and $j$ can transmit at the same time.

In TDMA, energy consumption can be reduced by eliminating collisions, avoiding idle listening, or entering inactive states (sleep mode) until their allocated time slots. The work in [1] proposes TDMA Mac protocol scheduling for many-to-one communication in wireless sensor network to reduce the delay and provide realtime guarantees of parallel transmission as well as save power by eliminating collisions [1].

The significance of this study is to fulfill the purpose mentioned above by applying Evolutionary Algorithm (EA) accompanied with Particle Swarm Optimization (PSO) Algorithm in MAC protocol (hybrid algorithm). The framework of PSO based on optimizing the total time for collecting a set of data and saving the energy consumed in switching between active and sleep states. In addition, this study concern of minimizing packet delay, maximizing parallel operation, minimizing number of collide packets and improve fairness, so that the overall network performance will be leveraged.

The rest of the paper is organized as follows: Section 2 presents some of the related works, Section 3 introduces the proposed scheme, Section 4 depicts simulation results and analysis, and finally, Section 5 concludes the paper and presents some future works.

\section{Literature Review}

On the bases of the above characteristics of WSNs discussed in section 1, a prominent method to avoid interference consists of adopting TDMA MAC protocols where neither collisions nor contention overhead are introduced [1]. Moreover, TDMA can guarantee a deterministic delay bound. Indeed, in TDMA protocols, time is divided into slots of equal length. Each slot is allocated to a node which can turn on the radio during the transmission of its data in the time slot allocated for it and turn off the radio when not transmitting. Since power consumption is one of the most important issues in WSN, such protocols can be used to save energy and sensor batteries' lifetime can be effectively managed and utilized. In this area, many works have been carried out. In fact, many research papers have been published on energy saving problem for TDMA protocols [2], [3].

In [4], Pantazis et al. proposed a TDMA scheduling algorithm to minimize power consumption by making an appropriate transmission schedule in the WSN. The aim of the proposed WakeUp intervals scheduling 
scheme is to achieve low level of energy consumption and to reduce the time delay from the sensors to the base station. The proposed scheme achieves the reduction of the end-to-end delay caused by the sleep mode operation while at the same time it maximizes the energy savings. Authors in [5] proposed a Minimum Delay Scheduling (MDS) algorithm and proved that using their approach the time delay will be reduced in clustered WSN. In fact MDS algorithm incorporates the concept of slot reuse which reduces significantly the end to end latency .

More recently, some researchers started investigating the usage of bio-inspired algorithms on intelligent optimizations of WSNs [6]. Such networks has significant similarities with biological systems or colonies, and as a consequence biologically-inspired approaches can be considered as promising references due to the fact that they are highly capable of self-adaptation and especially evolution. In [7] and under the framework of evolutionary search algorithms, Tao Wang et al. proposed a multi-objective PSO based on Pareto optimality algorithm to find a tradeoff between power consumption and time delay in WSN. In [8] authors proposed a multi-objective Artificial Immune System (AIS) Algorithm to find optimal solution to TDMA scheduling problem in wireless sensor networks.

Authors in [9] introduced a research paper related to improving network lifetime through energy consumption during node's transmission. Indeed, they proposed a genetic algorithm (GA) based approach where the relay nodes can form a cluster to route data to the sink node and an efficient scheduling for data gathering of relay nodes, and showed that this will minimize energy consumption of the relay nodes. They proved that, in a sensor network with a limited number of nodes where the global optimum can be determined, their proposed approach is capable to reach the optimal solution.

Yuhui shi et al. discussed, in their empirical study [10], the fast convergence of particle swarm optimization (a global optimization meta- heuristic designed for continuous problems) towards the optimal solutions under the different testing cases and also noticed that the speed of convergence near the minimum is becoming slower and proposed an adaptive inertia weight approach to ameliorate the convergence speed of PSO near the optimum.

\section{Methodology}

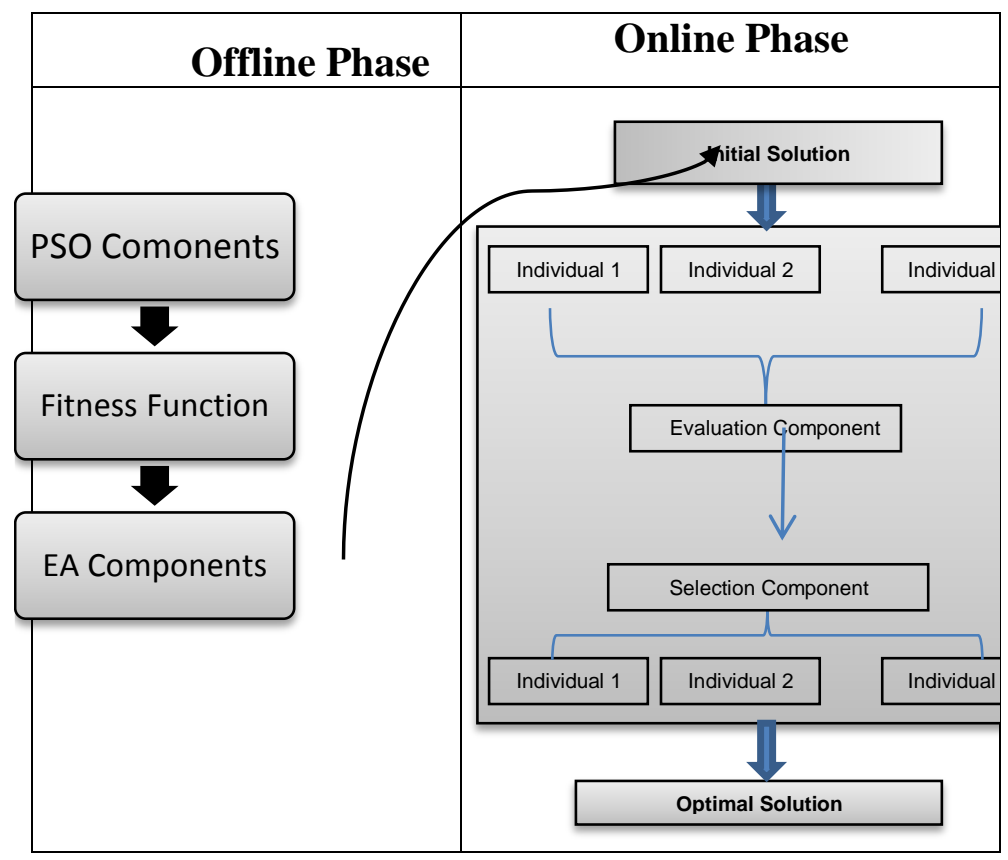

Fig. 1. The proposed algorithm. 
The main goal of this paper is to optimize both the total time spent in transmission and the energy consumption by switching between the active and sleep states. Other goals were also considered such as: packet delay, parallel transmissions, number of collided packets. The framework for the proposed hybrid algorithm is described as shown in Fig. 1. The result of these steps is dividing the population into many clusters called population individual. The process of encoding and decoding are key steps to use evolutionary algorithm. In encoding, the solution is described by a sequence code that is typically representing one individual in population-based algorithms. At first, we denote a Concurrent-Transmission as (PID, SEQ_NO), where PID identifies which transmission cycle the Concurrent-Transmission belongs to, and SEQ_NO is the sequence number of Concurrent-Transmission PID in all Concurrent-Transmissions.

The following example illustrates the proposed encoding mechanism. In this example, we have 2 transmission cycles: the first cycle is transmitting a packet from node 0 to $\mathrm{AP}$, and the second cycle is transmitting a packet from node 1 to AP. Each cycle consists of 4 concurrent transmissions. For the first cycle we have the following: $\{(0,0),(0,1),(0,2)$, and $(0,3)\}$. And for the second cycle we have the following: $\{(1$, $0),(1,1),(1,2)$, and $(1,3)\}$. One can generate a random combination of above concurrent transmissions, such as $(1,0)(0,0)(0,1)(0,2)(1,1)(0,3)(1,2)$ and $(1,3)$. A possible encoding for the above sequence is by eliminating the PTIDs out, hence we get the following encoding: 10001011. In the decoding step, we reverse the operation and transform concurrent transmissions to individuals. Slots are assigned from the first to the last concurrent transmission respectively at the same time. This assignment of concurrent transmissions to one slot is done without rousing collisions, as follows [1]:

\begin{tabular}{|c|c|c|c|c|c|c|c|}
\hline Slot No. & 1 & 2 & 3 & 4 & 5 & 6 & 7 \\
\hline concurrent transmission & $(0,0)$ & $(0,1)$ & $(0,2)$ & $(0,3)(1,0)$ & $(1,1)$ & $(1,2)$ & $(1,3)$ \\
\hline Execution node & 0 & 2 & 3 & 4,1 & 2 & 3 & 4 \\
\hline
\end{tabular}

Fig. 2. Slot allocation.

Now, it is obvious that an individual can be decoded to a slot scheduling with parallel operations. To evaluate the quality of a solution, both PSO and EA algorithms use fitness function. In PSO, Each cluster has a number of fitness functions and is computed using the following formula [1]:

$$
F_{i t}(i)=F(s)=\alpha * \text { Total_Energy }+(1-\alpha) * \text { Totalslots }
$$

where, $i$ : represents an individual of the evolution algorithms.

$s$ : refers to TDMA schedule and is decoded from individual $i$.

Total_Energy: is the total energy consumption.

TotalSlots: is the total number of slots under the schedule.

$\alpha$ : is a tradeoff factor that favors between Total_Energy and TotalSlots.

Moreover, the same formula used in EA to get the fitness function from all populations. In addition, Evolutionary algorithm based on 2 basic steps to improve PSO results, which are:

- Applying the core operators including selection (exploitation), and crossover (exploration). In which it selects the best candidate of the population according to the fitness function.

- EA depends on the trade-off between exploitation and exploration.

The fitness functions used in EA are: Schaffer f6, Sphere, and Generalized Griewank [5]. There is a tradeoff for using these functions, because $\mathrm{f} 6$ and Griewank suffer from local minima problem while sphere has no local minima, in the contrary, mean error for $\mathrm{f6}$ is less than 0.000001 , while in sphere the mean error is less than 0.01 . 


\section{Offline Phase}

We run the hybrid algorithm with Schaffer f6, Generalized Griewank, and Sphere with swarm size=population size $=30$. In addition, run the PSO part $(1,5,10,15,20,100)$ times. Using $C 1=C 2=2, W=1.5$, Pmutation and Pcrossover values are 0.4 and 0.8 respectively. The used parameters are listed in Table 1.

Table 1. Simulation Parameters, Initial Report

\begin{tabular}{ll}
\hline \multicolumn{1}{c}{ Parameter } & \multicolumn{1}{c}{ value } \\
\hline Tournament Selection Used & 2 \\
Crossover Strategy & 1 existed with swapping \\
Population size & 30 \\
Total no. of generations & 5 \\
Cross over probability & 0.4 and 0.8 \\
Number of variables & 30 \\
Total Runs to be performed & 5 \\
Exponent (n for SBX) & 2.00 \\
\hline \hline
\end{tabular}

The result of applying the hybrid algorithm for this sample is a file contains a number of fitness functions for each cluster, and then this file is taken as input for EA to gain the best fitness function for each cluster. Table 2 presents the results of applying the PSO algorithm, which in turn is feed to the EA algorithm as described above.

Table 2. The Result of Applying Hybrid Algorithm

\begin{tabular}{|l|l|l|l|l|}
\hline 0.805443 & 0.917799 & 0.853510 & 0.716384 & 0.775796 \\
\hline 0.749848 & 0.960530 & 0.816520 & 0.962443 & 0.918643 \\
\hline 0.958651 & 0.759272 & 0.962272 & 0.902580 & 0.910018 \\
\hline 0.716815 & 0.868530 & 0.824196 & 0.815932 & 0.848522 \\
\hline 0.916097 & 0.824932 & 0.914898 & 0.921036 & 0.862142 \\
\hline 0.913116 & 0.858790 & 0.921438 & 0.960261 & 0.924349 \\
\hline
\end{tabular}

Feeding these values to the EA component and apply the hybrid algorithm results are depicted in Table 3. It displays each run and the best fitness function for each run and for each individual.

Table 3. Obtained Results for All Runs

\begin{tabular}{|c|c|c|c|c|c|}
\hline Run Number & 1 & 2 & 3 & 4 & 5 \\
\hline Max & 0.01595 & 0.01803 & 0.01898 & 0.01842 & 0.01802 \\
\hline Min & 0.02162 & 0.02239 & 0.02387 & 0.02199 & 0.02079 \\
\hline Average & 0.01973 & 0.02045 & 0.02194 & 0.02152 & 0.02058 \\
\hline No. of mutations & 52 & 72 & 62 & 61 & 75 \\
\hline No. of x-overs & 60 & 59 & 59 & 57 & 57 \\
\hline Best ever & 1.785631 & 1.411144 & 1.631983 & 1.649751 & 1.642827 \\
\hline Fitness & 0.021621 & 0.022389 & 0.023867 & 0.021990 & 0.020791 \\
\hline Best individual & 1.785631 & 1.411144 & 1.631983 & 1.649751 & 1.642827 \\
\hline
\end{tabular}

\section{Simulation Results}

An event-based simulation is used to perform the evaluation study. The simulator is developed in C++ environment. Several performance metrics were used to evaluate the performance of the proposed method as follows: number of time slots reserved to finish a sampling round, total energy consumption of the 
network to finish a sampling round, and slot utility. We aim to minimize the total energy consumption in order to extend network lifetime. The slot utility is the number of empty slot divided by the number of total slots, and it describes the channel efficiency. Fig. 3 and 4 show a comparison between PSO and HA before changing the adaptation (before applying fitness functions f6, Sphere and Griewank).

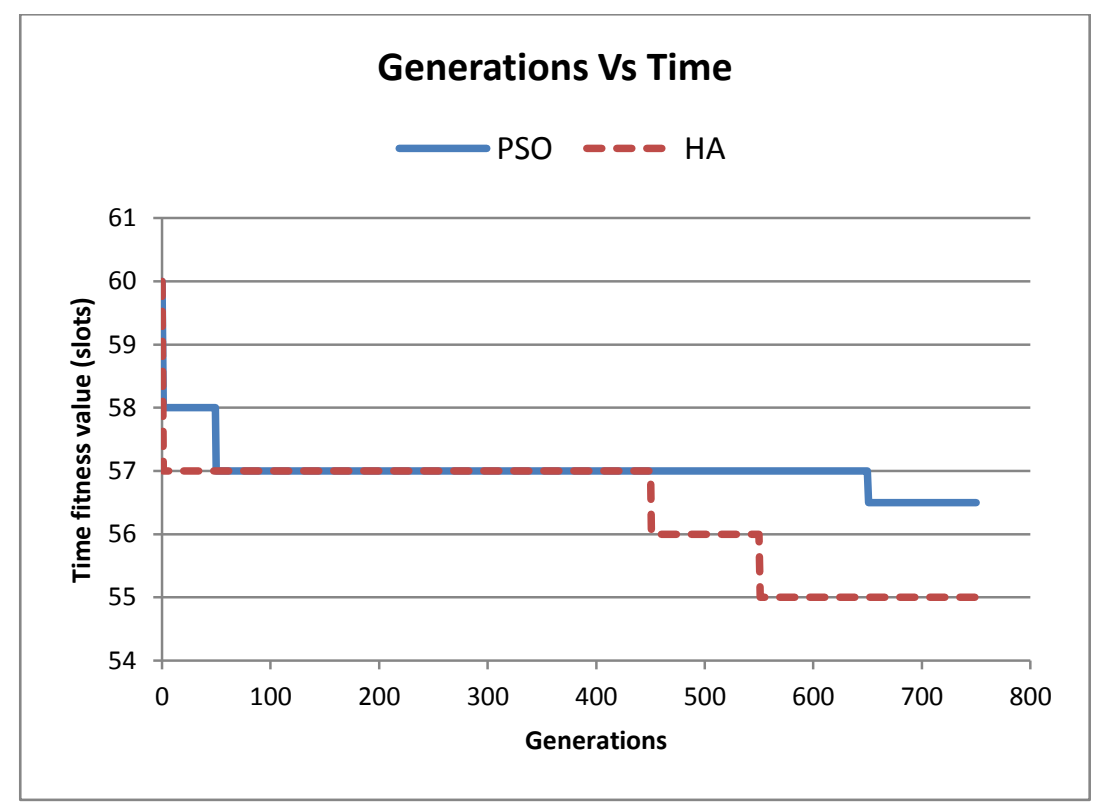

Fig. 3. Time performance over generations.

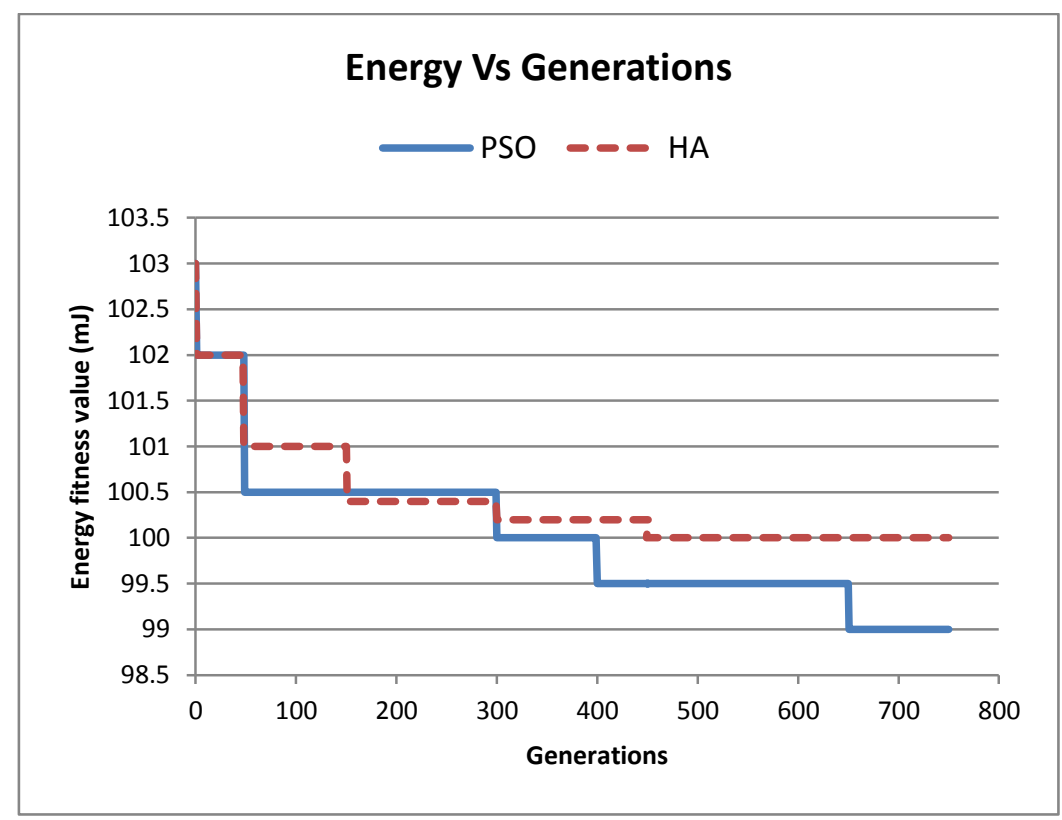

Fig. 4. Energy performance over generations.

Fig. 3 shows the time fitness value in term of slots over generation for both HA and PSO. As we can see that both PSO and HA decrease from fitness value of 60 to around 57 during the initial improvements they achieve. However, HA drops much faster within only few generations which PSO takes around 50 generations to converge to the same value of 57. After that both algorithms remains constant until around 450 generations for HA and around 650 for PSO after which they will have another improvements. HA performs 
better and converges to value of 55 after 550 generations which PSO remains around the value 56.5 after 650 generations.

Fig. 4 shows the energy fitness value over generation for both HA and PSO. We can observe a bit similar behavior with more drops and improvements every 50 to 100 generations. The initial value for both techniques was 103 and it converges to value of 99 after 650 generations while HS converges to the value 100 within 450 generations. In the following two figures we show a comparison between Schaffer, Sphere and Griewank in term of fitness and time versus the number of runs.

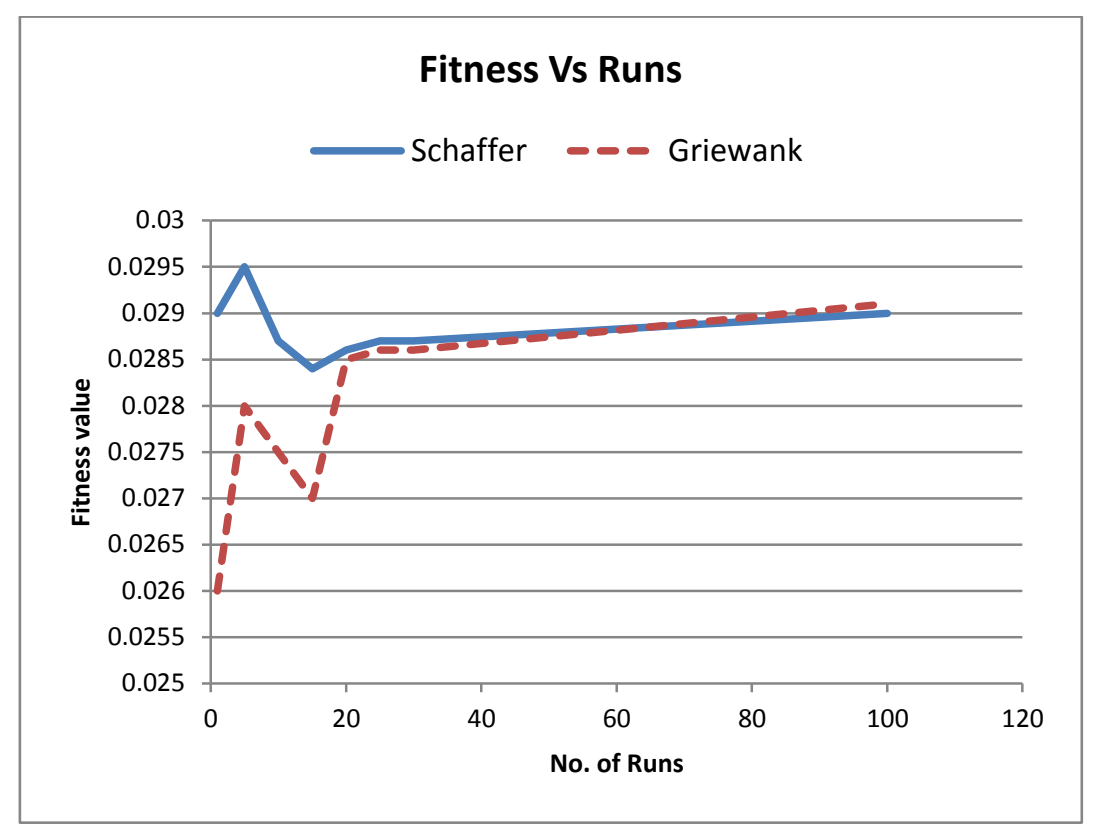

Fig. 5. Fitness value over number of runs.

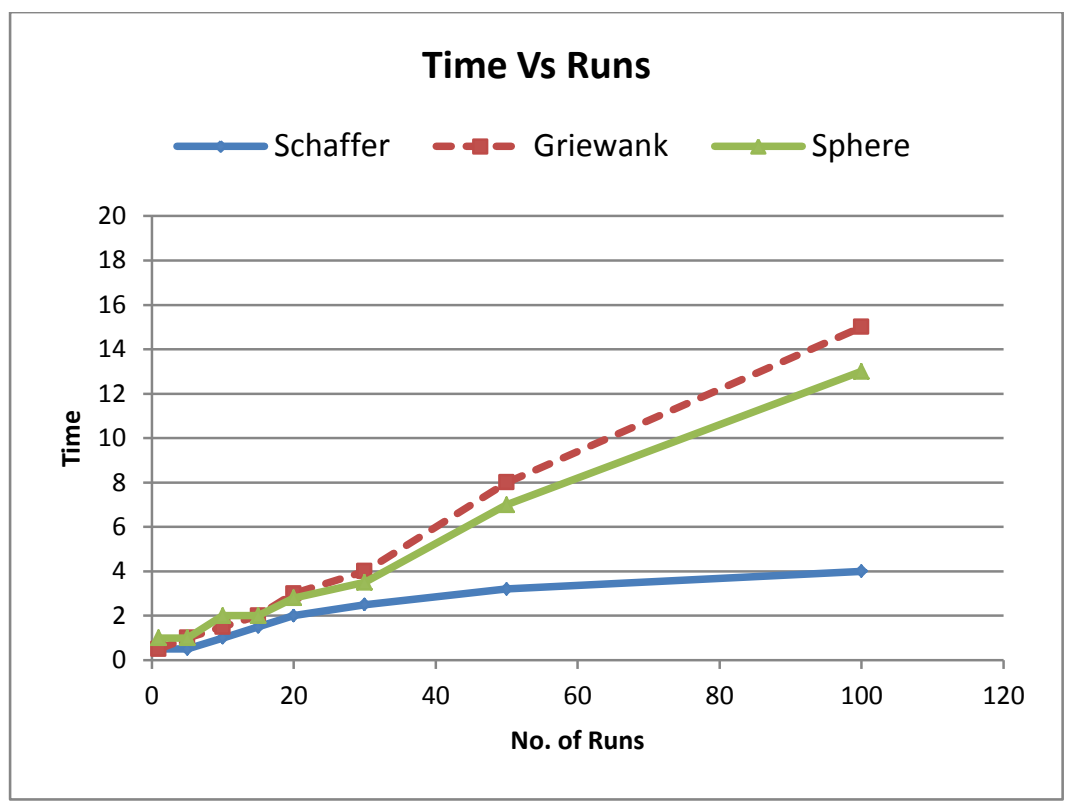

Fig. 6. Time over number of runs.

Fig. 5 summarize the relation between fitness values over number of runs, it shows that Schaffer gives better fitness values than the Griewank for small number of runs, But Griewank give better results than 
Schaffer after the number of runs is above 100. Fig. 6 shows that Schaffer needs much less time than both Griewank and Sphere which both have similar behavior with better value for Sphere. This will improves the total performance of the network when using Schaffer rather than Griewank or Sphere.

\section{Conclusion}

The problem of time scheduling in TDMA is a crucial one to improve the network lifetime. In this paper we have discussed TDMA scheduling in many-to-one sensor networks. Then we proposed a hybrid a scheduling algorithm that employee both PSO and EA algorithms using different optimization functions to reduce the delay time and provide real-time guarantees of parallel transmission as well as save power by eliminating collision. The simulation results demonstrated that the PSO different optimization functions would give different fitness values and time. Schaffer gives better performance in terms of time; however it suffers from the local minima problem.

\section{References}

[1] Ye, W., John, H., \& Deborah, E. (2002). An energy-efficient MAC protocol for wireless sensor networks. Proceedings of the 21st Annual Joint Conference of the IEEE Computer and Communications Societies: Vol. 3.

[2] Ergen, S., \& Pravin, V. (2010). TDMA scheduling algorithms for wireless sensor networks. Wireless Networks, 16(4), 985-997.

[3] Xu, N. (2002). A survey of sensor network applications. IEEE Communications Magazine, 40(8), 102-114.

[4] Pantazis, N. (2009). Energy efficiency in wireless sensor networks using sleep mode TDMA scheduling. Ad Hoc Networks, 7(2), 322-343.

[5] Shi, L., \& Abraham, F. (2010). TDMA scheduling with optimized energy efficiency and minimum delay in clustered wireless sensor networks. Mobile Computing, 9(7), 927-940.

[6] Jabbar, S. (2013). Intelligent optimization of wireless sensor networks through bio-inspired computing: Survey and future directions. International Journal of Distributed Sensor Networks.

[7] Wang, T., Zhiming, W., \& Jianlin, M. (2007). A new method for multi-objective tdma scheduling in wireless sensor networks using pareto-based pso and fuzzy comprehensive judgement. High Performance Computing and Communications, 144-155.

[8] Davarzani, Z., Mohammah, Y., \& Mohammad, A. (2011).TDMA scheduling in wireless sensor network using artificial immune system. Soft Computing in Industrial Applications, 175-185.

[9] Bari, A. (2009). A genetic algorithm based approach for energy efficient routing in two-tiered sensor networks. Ad Hoc Networks, 7(4), 665-676.

[10] Shi, Y., \& Russell, C. (1999). Empirical study of particle swarm optimization. Proceedings of the 1999 Congress on Evolutionary Computation: Vol. 3.

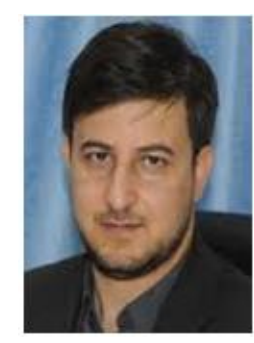

Yaser Khamayseh is the associate professor at Jordan University of Science and Technology. He received the B.Sc. degree in computer science from Yarmouk University, Jordan in 1998, and M.Sc. from University of New Brunswick, Canada in 2001. He received the Ph.D. degree in computer science in 2007 from University of Alberta, Edmonton, Canada. In 2007 he joined the faculty of computer and information technology at Jordan University of Science and Technology, Jordan, as an assistant professor of computer science. He has served as a technical program committee member of various conferences.

His research interests include wireless network optimization, resource management in wireless networks, and protocol design and analysis for future generation wireless communication networks and systems. 


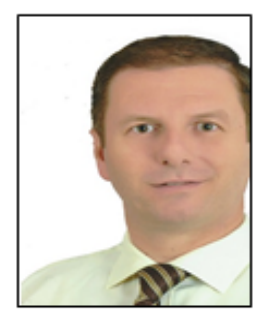

Wail Mardini is an assistant professor at the Computer Science department at Jordan University of Science and Technology (JUST)/Jordan. He received his B.Sc in applied mathematics from JUST in 1997, and received the master's degree in computer science from University of New Brunswick/Canada in 2001, and received his Ph.D. degree in computer science from University of Ottawa/Canada in 2006. His research interests include the following areas: wireless mesh networks, wireless sensor networks, wireless optical mesh networks, optical network survivability, wimax technology, scheduling in parallel computing and intrusion detection in data base techniques.

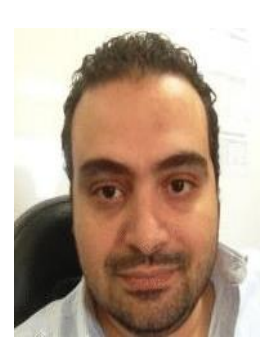

Nadhir Ben Halima received his B.S. degree in computer engineering from the National School of Computer Sciences (ENSI), Manouba, Tunisia, the M.S degree in communication networks engineering from Sant'Anna School of Advanced Studies, Pisa, Italy, in 2006, and the Ph.D. degree in information and communication technology in 2009 from the University of Trento, Trento, Italy. In 2009 he was a visiting researcher at the Department of Electrical and Computer Engineering at North Carolina State University, Raleigh, NC, USA. Since September 2011, he is the assistant professor and the vice dean of the College of Computer Science and Engineering at Taibah University, Yanbu Branch, Yanbu, Saudi Arabia. His research interests include wireless and sensor networks, cognitive networks, image watermarking, and multirobot systems. 